\title{
Massachusetts General Hospital and the Shanghai Mental Health Center: the past, present and future of a psychiatric research partnership
}

\author{
Tristan Barako (D , ${ }^{1}$ Chunbo Li, ${ }^{2}$ Albert Yeung ${ }^{3,4}$
}

\section{INTRODUCTION}

There are many obstacles to developing, maintaining and strengthening international research collaborations, whether they are posed by geography, culture or, most recently, global economics and politics. It is critically important to overcome these obstacles, especially when it comes to fostering the types of breakthrough biomedical science that benefit from diverse perspectives, methodologies and data sets. Moreover, the research underlying these breakthroughs unfolds over the course of years, and even decades. For these reasons, and more, the Department of Psychiatry at Massachusetts General Hospital (Mass General) and the Shanghai Mental Health Center (SMHC) are taking the long view: exploring and implementing numerous collaborative research and training projects that are leading to better care and treatments for patients, both in the USA and China, who suffer from a range of psychiatric disorders. We hope that our experiences can serve as a model for other institutions seeking to forge international partnerships focused on medical care, research and training.

\section{BACKGROUND HISTORY}

Founded in 1811, Mass General remains at the forefront of medicine, fostering a culture of collaboration,

\footnotetext{
'Development, Massachusetts General Hospital, Boston, Massachusetts, USA

${ }^{2}$ Shanghai Key Laboratory of Psychotic Disorders, Shanghai Mental Health Center, Shanghai Jiao Tong University School of Medicine, Shanghai, China ${ }^{3}$ Psychiatry, Massachusetts General Hospital, Boston, Massachusetts, USA

${ }^{4}$ Psychiatry, Harvard Medical School, Boston,

Massachusetts, USA

Correspondence to Dr Tristan Barako; tbarako@mgh.harvard.edu
}

pushing the boundaries of medical research and educating the brightest medical minds. Mass General has been ranked among the top five hospitals in the USA by US News $\mathcal{E}^{\circ}$ World Report ever since the rankings began-currently it stands at number 2-and is the only hospital ranked in all 16 specialties, a testament to the breadth and depth of the hospital's expertise. The original and largest teaching hospital of Harvard Medical School, Mass General is home to the largest hospital-based research programme in the USA, with an annual research budget of more than $\$ 1$ billion, spanning more than 30 clinical departments and centres across the hospital. On the strength of its research enterprise, Newsweek recently ranked Mass General the number 6 hospital worldwide.

When Mass General's Department of Psychiatry was founded in 1934, it was one of only three general hospital psychiatry services in the USA. For 20 of the last 25 years - and including the current year-US News E World Report has ranked Mass General number 1 in Psychiatry, based in large part on its 60 clinical and research programmes, over 500 faculty (including $300 \mathrm{PhDs}$ ) and 150 research staff. The Department's faculty are uniquely trained as clinicians, researchers and teachers, and include some of the field's most accomplished and recognised specialists, particularly in psychopharmacology, cognitive-behavioural therapy and behavioural medicine.

Mass General Psychiatry's Center for Anxiety and Traumatic Stress Disorders (CATSD) and Complicated Grief Program is especially strong in these approaches to care. CATSD's research portfolio is focused on better understanding the underlying causes, psychology and neurobiology of anxiety disorders-including panic disorder, social anxiety disorder, generalised anxiety disorder, phobias, complicated grief and post-traumatic stress disorder. The Center's leaders, Dr Jerrold Rosenbaum and Dr Luana Marques, along with CATSD faculty, postdoctoral fellows and colleagues, contributed the articles appearing in this issue.

The SMHC is a leading institution for mental healthcare, research and professional training in Shanghai and all of China. Of more than 1000 professional staff at SMHC providing care, there are 380 psychiatrists and 590 psychiatric nurses, with more than a third being senior leaders in the field, on the strength of their treating some of the most severe, acute and refractory mental illnesses (e.g., schizophrenia, affective disorder, psychosis, neurodegenerative disorders, substance abuse).

The bulk of SMHC's research is conducted at the Shanghai Institute of Mental Health (SIMH), which consists of 17 research departments, including biochemistry, genetics, neurophysiology, neuroimaging, traditional Chinese medicine, clinical epidemiology, psychiatric nursing, psychotherapy and public mental health. Research in these departments has earned SIMH staff numerous academic awards, particularly in geriatric and preventive psychiatry. Guided by a biopsychosocial model, which embraces a holistic approach to mental health, SIMH is the leading psychiatric clinical care and research institution in Shanghai, with aspirations to become also a leading mental health research centre globally. To this end, SMHC is a WHO collaborating centre for research and training in mental health, which has led to numerous international collaborations with psychiatrists worldwide, including with faculty at Mass General.

Connections between Mass General Psychiatry and SMHC have been forming for nearly 20 years. For example, in 2003, Dr Xu Yong and one of the coauthors of this article, AY, collaborated on a study on depression 
in primary care settings, in which they explored the use of screening instruments for early detection of depression. Their collaborative effort led to further research and published manuscripts from both institutions (e.g., refs 1 2). They followed up this work with a comparative study of sleep paralysis among American and Chinese patients. ${ }^{3}$

The groundwork for a formal partnership between Mass General Psychiatry and SMHC, however, was truly laid in 2007, when SMHC was hosting a meeting of the World Psychiatric Association. A delegation from Mass General, led by then Chair Dr Jerry Rosenbaum, and including Dr Bob Birnbaum, Dr Darin Dougherty, Dr Jonathan Worth and coauthor AY, visited Shanghai and was hosted by Dr Zeping Xiao, then president of the SMHC. Many fruitful discussions about the types and scope of collaboration were held during this visit, with a consensus forming around training. In the following year, 2008, the partnership was formalised through a Memorandum of Understanding, sponsored by Johnson \& Johnson China, with a focus on physician exchanges. More specifically, two SMHC physicians were chosen each year to spend several months at Mass General in the role of observers. The programme, which lasted 4 years, generated friendship, trust and scientific familiarity between both institutions, and laid the foundation for subsequent research collaborations. In particular, a joint team led by Dr Jijun Wang (SMHC) and Dr Dave Henderson (Mass General) studied metabolic syndromes in patients with schizophrenia, resulting in a series of articles in leading journals. ${ }^{45}$

\section{RECENT ACTIVITY AND FUTURE DIRECTIONS}

The Mass General-SMHC relationship was revitalised in 2018 thanks to a conference held in Shanghai, which was cohosted by Harvard, Mass General and SMHC, and cosponsored by the Harvard China Fund and the Tianqiao and Chrissy Chen Institute (TCCI). The objectives of

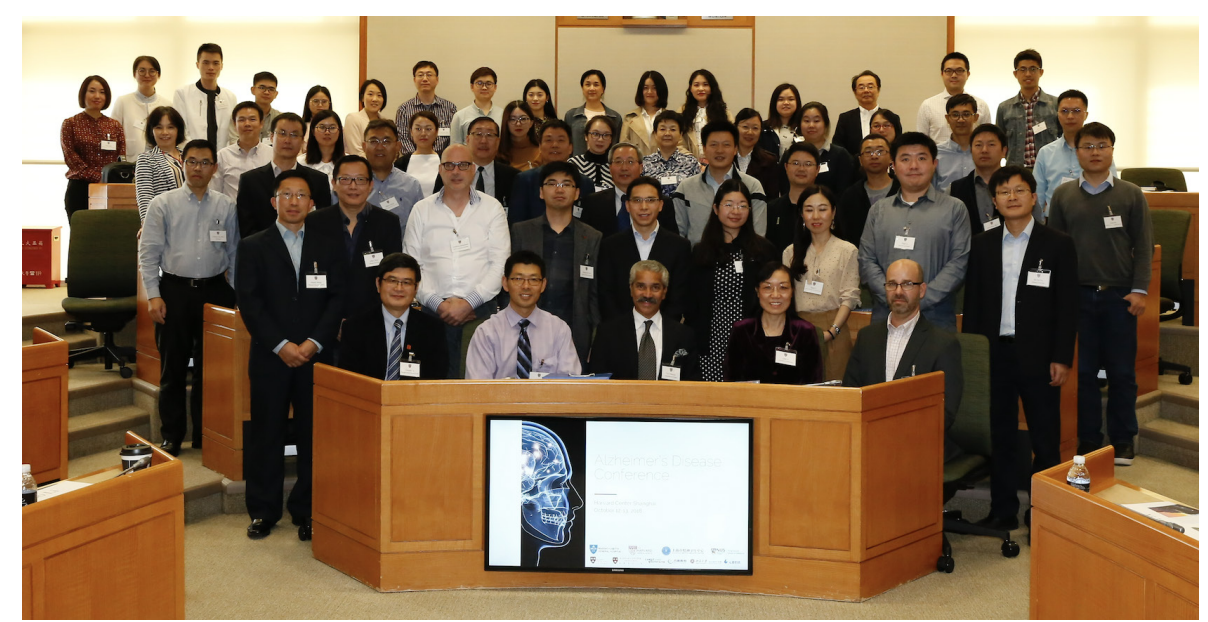

Figure 1 Harvard-Shanghai Conference on Brain Health - a special meeting for understanding and intervention of Alzheimer's disease, 12-13 October 2018. Seated (left to right): Chunbo Li, MD (Shanghai Mental Health Center, SMHC); Can Zhang, MD, PhD (Mass General); Sangram Sisodia, PhD (University of Chicago); Ling Li, $\mathrm{PhD}$ (University of Minnesota); Tristan Barako, PhD (Mass General).

the conference were to foster international academic exchangesparticularly between China and the USA-and cross-disciplinary communication and cooperation, all with the ultimate goal of hastening new translational approaches and broadening the scope of interventions for Alzheimer's disease. ${ }^{6}$ Dr Can Zhang (Mass General), Dr Chongzhao Ran (Mass General) and coauthor CL (SMHC) were among the organisers of this conference (see figure 1).

One of these objectives has already been achieved in that conversations and meetings between Mass General and SMHC have intensified over the past several months. Notable in this respect was a March visit to SMHC, hosted by President Yifeng $\mathrm{Xu}$, by a delegation from Mass General, led by Dr Tim Ferris, chief executive officer of the Massachusetts General Physicians Organization (see figure 2). The main outcome of this visit was a commitment to expand on the previous partnership, primarily through a slate of collaborative, translational research projects focused on a rigorous combination of traditional Chinese and modern Western medicine, as well as well-integrated junior physician and nurse training, all supported by state-of-the-art infrastructure. Also catalysing these conversations is SMHC's recent (June 2018) integration into the TCCI for
Translational Research, whose goals of better understanding, preventing and treating mental illness are well aligned with those of Mass General Psychiatry.

\section{DISCUSSION}

Psychiatric disorders are unconstrained by international boundaries, as evidenced by comparable incidence rates for many of these disorders in China and the USA. Correspondingly, preventing and treating these disorders requires a global effort-one based on the open exchange (within the applicable legal and regulatory frameworks) of data, ideas, methodologies and expertise, many of which are complementary and synergistic. Moreover, collaborations between non-profit medical institutions are driven primarily by a desire to alleviate human suffering. To this end, patient outcomes and research results often quickly enter the public domain, through presentations at conferences and publications in peer-reviewed journals, by which means they have the potential to do the greatest good. Such collaborations, as between Mass General and SMHC, can serve as models for other international partnerships, perhaps even those involving the public and for-profit sectors. Models of successful partnership become all 


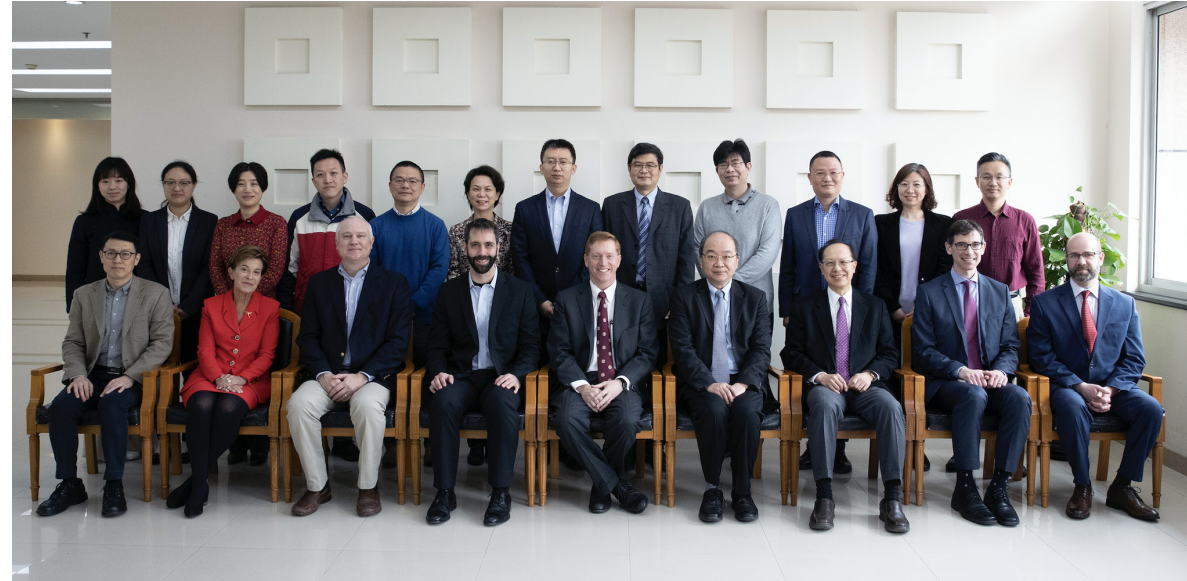

Figure 2 Mass General visit to Shanghai Mental Health Center (SMHC), 20 March 2019. Seated (left to right) (Mass General unless noted otherwise): Bin Xie, MD (Party Secretary, SMHC); Jeanette Ives-Erickson, RN, DNP (Chief Nurse Emerita); Darin Dougherty, MD (Director, Division of Neurotherapeutics); Jacob Hooker, $\mathrm{PhD}$ (Associate in Neuroscience, Martinos Center); Timothy Ferris, MD (CEO, Mass General Physicians Organization); Yifeng Xu, MD (President, SMHC); Albert Yeung, MD (Director, Clinical Studies, Psychiatry); Michael McNally (Vice President, Development); Tristan Barako, PhD (Senior Director, Development). Standing (left to right) (all SMHC): Jiayan Wu, MSc (Director of International Affairs); Ying Lu, MD (Attending doctor, Division of Traditional Chinese Medicine); Min Zhao, MD (Vice President); Fuzhong Yang, MD (Attending doctor, Division of Clinical Psychiatry); Daihui Peng, MD (Director, Division of Mood Disorder); Yanling He, MD (Director, Division of Health Education and Health Promotion); Zhen Wang, MD (Vice President); Chunbo Li, MD (Vice President); Yang Shao, MD (Vice President); Yifeng Shen, MD (Vice Director, Division of Clinical Research Center Office); Jue Chen, MD (Director, Division of Psychosomatic Medicine); Qing Zhang, MSc (Vice Director, Division of Research Management and Educational Office).

the more important when the obstacles opposing them seemingly grow in size and number. There is, however, too much at stake-the mental health of countless patients in China, the USA and elsewhere-not to overcome them.

Funding The authors have not declared a specific grant for this research from any funding agency in the public, commercial or not-for-profit sectors.

Competing interests None declared.
Patient consent for publication Not required.

Provenance and peer review Not commissioned; externally peer reviewed.

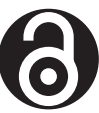

\section{OPEN ACCESS}

Open access This is an open access article distributed in accordance with the Creative Commons Attribution Non Commercial (CC BY-NC 4.0) license, which permits others to distribute, remix, adapt, build upon this work non-commercially, and license their derivative works on different terms, provided the original work is properly cited, appropriate credit is given, any changes made indicated, and the use is non-commercial. See: http://creativecommons.org/licenses/by-nc/4.0/.

(C) Author(s) (or their employer(s)) 2019. Re-use permitted under CC BY-NC. No commercial re-use. See rights and permissions. Published by BMJ.

\section{Check for updates}

To cite Barako T, Li C, Yeung A. General Psychiatry Epub ahead of print: [please include Day Month Year]. doi:10.1136/gpsych-2019-100157

Received 4 October 2019

Revised 18 October 2019

Accepted 23 October 2019

General Psychiatry 2019;32:e100157.

doi:10.1136/gpsych-2019-100157

ORCID iD

Tristan Barako http://orcid.org/0000-0002-6694-3760

\section{REFERENCES}

1 Yeung A, Kung WW, Murakami JL, et al. Outcomes of recognizing depressed Chinese American patients in primary care. Int $\mathrm{J}$ Psychiatry Med 2005;35:213-24.

2 XU Y, HS W, YF X. The reliability and validity of patient health questionnaire depression module (PHQ-9) in Chinese elderly. Shanghai Archives of Psychiatry 2007;19:257-9. Chinese.

3 Yeung A, Xu Y, Chang DF. Prevalence and illness beliefs of sleep paralysis among Chinese psychiatric patients in China and the United States. Transcult Psychiatry 2005:42:135-45.

4 Wang J, Liu EY, Freudenreich O, et al. Phenotypic characteristics in metabolically obese but normal weight non-diabetic patients with schizophrenia. Schizophr Res 2010;124:49-53.

5 Yi Z, Fan X, Wang J, et al. Rosiglitazone and cognitive function in clozapine-treated patients with schizophrenia: a pilot study. Psychiatry Res 2012;200:79-82.

6 Zhang C, Lu Y, Feng L, et al. Proceedings of the Harvard-Shanghai Conference on Brain Health - A special meeting for Understanding and Intervention of Alzheimer's Disease. Journal of Advances in Health 2019;1:1-8.

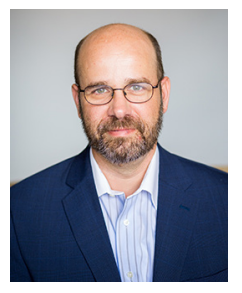

Dr.Tristan Barako is the senior director of development for foundation fundraising in the neurosciences at Massachusetts General Hospital. He has broad experience as a fundraiser, writer, and researcher in diverse settings, especially health care, higher education, and media production. Previously he served as the senior research development specialist in Brown University's Office of the Vice President for Research, focusing on federal funding. Before working in fundraising, as senior writer and researcher at Providence Pictures, he researched, wrote, and edited scripts for highly-rated, award-winning documentaries appearing on America Public Television (PBS/NOVA). Dr. Barako trained as an archaeologist, receiving his doctorate and masters degrees at Harvard University, during which time he excavated and surveyed throughout the Mediterranean region, both on land and at sea. He has more than a dozen publications to his name in a variety of fields, including multiple articles and reviews in leading journals, as well as two monographs. 